
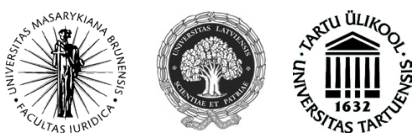

ISSN 1392-6195 (print) ISSN 2029-2058 (online) JURISPRUDENCIJA JURISPRUDENCE 2014, 21(1), p. 59-88.

\title{
DER EUROPÄISCHE MINDERHEITENBEGRIFF ENTWICKLUNG UND WIRKUNG Teil II
}

\author{
Arndt Künnecke \\ MEF University Istanbul \\ Ulus Mah., Öztopuz Cad., Leylak Sok., \\ 34340 Ulus-Besiktas, Turkey \\ Phone: 00905378334566 \\ E-mail: kuennecke@web.de
}

Received on 20 September 2013, accepted on 13 March 2014

doi:10.13165/JUR-14-21-1-04

Diese Publikation ist die Fortsetzung des gleichnamigen Aufsatzes aus der vorigen Jurisprudencija-Ausgabe vom 2013, 20(4).

Nachdem im ersten Teil der Untersuchung die Entwicklung des Minderheitenbegriffs auf internationaler Ebene in der UN, der UNESCO und der KSZE/OSZE dargestellt und dabei festgestellt wurde, dass es keine allgemein anerkannte und völkerrechtlich verbindliche Minderheitendefinition gibt, werden nun im zweiten Teil der Untersuchung anhand von Dokumenten des Europarats und der EG/EU die Kriterien des europäischen Minderheitenbegriffs herausgearbeitet sowie die daraus erwachsenden Minderheitenrechte aufgezeigt, die den unter den europäischen Minderheitenbegriff fallenden Gruppen in Europa zugestanden werden.

\subsubsection{Europarat}

Der am 5.5.1949 in London gegründete Europarat mit Sitz in Straßburg ist die älteste „europäische“ Organisation. Seine Mitglieder verpflichteten sich zum

Jurisprudencija/Jurisprudence

(C) Mykolo Romerio universitetas, 2014

(C) Mykolas Romeris University, 2014
ISSN 1392-6195 (print), ISSN 2029-2058 (online) http://www.mruni.eu/lt/mokslo_darbai/jurisprudencija/ http://www.mruni.eu/en/mokslo_darbai/jurisprudencija/ 
Schutz und zur Förderung gemeinsamer Grundsätze und Ideale. Als eines seiner wichtigsten Ziele hat sich der Europarat dabei den Schutz der Menschenrechte und Grundfreiheiten sowie die Vorherrschaft des Rechts gesetzt. ${ }^{1}$

Bereits seit seiner Gründung versteht sich der Europarat als „Wächter der Menschenrechte“2. Mit der Europäischen Konvention zum Schutz der Menschenrechte und Grundfreiheiten (EMRK) von 1950 und deren rechtlichem Kontrollmechanismus durch die Europäische Menschenrechtskommission und den Europäischen Gerichtshof für Menschenrechte (EGMR) ${ }^{3}$ hat der Europarat den Schutz der Menschen- und Minderheitenrechte in seinen Mitgliedsstaaten am effektivsten garantiert und gefördert. ${ }^{4}$ Nach eigenem Bekunden des Europarates ist die Beachtung der Rechte der Minderheiten materielle Voraussetzung für den Beitritt neuer Mitglieder zum Europarat. ${ }^{5}$ Nach Inkrafttreten des Vertrages von Lissabon ist in Art. 6 II 1 EU-Vertrag nun auch der Beitritt der EU zur EMRK vorgesehen. Die Modalitäten des Beitritts werden derzeit zwischen Europarat und EU verhandelt. ${ }^{6}$

Im Rahmen der Arbeiten des Europarates lassen sich zwei Arten von Dokumenten unterscheiden: zum einen Dokumente, die Minderheitenschutzrechte enthalten, den Begriff der Minderheit jedoch nicht definieren, zum anderen Dokumente, die zwar eine Definition des Minderheitenbegriffs enthalten, aber nie das Entwurfsstadium überschritten haben. ${ }^{7}$

1 Vgl. Schmuck, O. Motive, Leitbilder und Etappen der Integration, in: Bundeszentrale für politische Bildung (Hrsg.). Informationen zur politischen Bildung 279, Europäische Union. Bonn, 2003: S. 12; Strasser, K. Grundrechtsschutz in Europa und der Beitritt der Europäischen Gemeinschaften zur Europäischen Menschenrechtskonvention. Frankfurt a. M., 2001: S. 106; White, N. D. The law of international organisations, 2nd Ed. Manchester, 2005: S. $132 \mathrm{f}$.

2 Steiner, L. Die Entwicklung des Minderheitenschutzes im Rahmen des Europarates, in: Blumenwitz, D.; Gornig, G. H. (Hrsg.) Minderheiten- und Volksgruppenrechte in Theorie und Praxis. Köln, 1993: S. 29.

3 Mit dem Inkrafttreten des 11. Protokolls zur EMRK am 1.11.1998 wurde dieser ZweikammerMechanismus dadurch ersetzt, dass der Europäische Gerichtshof für Menschenrechte (EGMR) seitdem die Funktion als Kontrollorgan in Sachen Menschenrechts- und Minderheitenschutz allein wahrnimmt (vgl. Art. 1 des 11. Protokolls zur EMRK, Text siehe: BGBl. 1995 II, S. 579591).

4 Vgl. Klebes, H. Rechtsschutz von Minderheiten - $\mathrm{Zu}$ den Arbeiten des Europarats, in: Blumenwitz, D.; Mangoldt, H. von (Hrsg.) Fortentwicklung des Minderheitenschutzes und der Volksgruppenrechte in Europa. Köln, 1992: S. 47; Schumann, K. Der Stand der Arbeiten des Europarates zu Fragen nationaler Minderheiten, in: Heuberger, V.; Suppan, A. (Hrsg.) Brennpunkt Osteuropa. München, 1996: S. 298 f.

5 Vgl. Richtlinie 484 (1993), in: Bundestags-Drs. 12/4572: S. 16.

6 Vgl. http://www.auswaertiges-amt.de/sid_0B5DF76BFA250E144FDD468AE5E9FF6E/DE/ Aussenpolitik/ Friedenspolitik/Europarat/ER_Text_node.html [aufgerufen am 18.8.2013].

7 Vgl. Gornig, G. H. Die Definition des Minderheitenbegriffs aus historisch-völkischer Sicht, in: Blumenwitz, D.; Gornig, G. H.; Murswiek, D. (Hrsg.) Ein Jahrhundert Minderheiten- und Volksgruppenschutz. Köln, 2001: S. 30. 
Vom Europarat ausgearbeitete völkerrechtliche Verträge sind - im Gegensatz zu den unverbindlichen KSZE/OSZE-Dokumenten - nach der Annahme und Ratifikation durch die Mitgliedsstaaten des Europarates für diese rechtlich verbindlich. $^{8}$ Das Organ, welches die Einhaltung der EMRK überwacht und sicherstellt, ist der Europäische Gerichtshof für Menschenrechte (EGMR). Gerade wegen dieser rechtlichen Verbindlichkeit kommt den ratifizierten Dokumenten des Europarates für den Minderheitenschutz in Europa besondere Bedeutung zu.

\subsubsection{Ratifizierte Minderheitenschutzverträge im Rahmen des Europarates}

\subsection{Europäische Menschenrechtskonvention (EMRK) von 1950}

Die Europäische Menschenrechtskonvention vom $5.11 .1950^{9}$ enthält weder selbst noch in einem der später in Ergänzung zu ihr in Kraft getretenen Zusatzprotokolle eine ausdrückliche Bestimmung zum Minderheitenschutz. Lediglich im Rahmen des Diskriminierungsverbots des Art. 14 EMRK findet der Begriff „,nationale Minderheit“ Erwähnung:

„Der Genuss der in dieser Konvention anerkannten Rechte und Freiheiten ist ohne Diskriminierung insbesondere wegen des Geschlechts, der Rasse, der Hautfarbe, der Sprache, der Religion, der politischen oder sonstigen Anschauung, der nationalen oder sozialen Herkunft, der Zugehörigkeit zu einer nationalen Minderheit ${ }^{10}$, des Vermögens, der Geburt oder eines sonstigen Status zu gewähren." ${ }^{11}$

Nach Art. 14 EMRK ist der Genuss der in der EMRK festgeschriebenen Rechte und Freiheiten somit ohne Benachteiligung $\mathrm{u}$. a. wegen der Zugehörigkeit zu einer nationalen Gruppe zu gewährleisten. Gleichwohl entfaltet Art. 14 EMRK nur begrenzte Schutzwirkung zugunsten nationaler Minderheiten, da diese Vorschrift nach der Rechtsprechung des EGMR lediglich ein Willkürverbot und gerade kein absolutes Verbot der Ungleichbehandlung aufgrund der in Art. 14 EMRK genannten Unterscheidungsmerkmale enthält. ${ }^{12}$ Unter dem Begriff „Minderheit“ in Art. 14 EMRK dürfen nach der Rechtsprechung des EGMR auch nur „nationale

8 Vgl. Steiner, L.: S. 30; Streinz, R. Minderheiten- und Volksgruppenrechte in der Europäischen Union, in: Blumenwitz, D.; Gornig, G. H. (Hrsg.) Der Schutz von Minderheiten und Volksgruppen durch die Europäische Union. Köln, 1996: S. 21.

9 UNTS Bd. 213: S. 221 ff. bzw. BGBl. 1952 II: S. 686 ff.

10 Textliche Hervorhebung des Autors.

11 UNTS Bd. 213: S. 232 f. bzw. BGBl. 1952 II: S. 690 f.

12 Vgl. EGMRE 2, 30 [unter I. B., \$ 10]. 
Minderheiten" verstanden werden. ${ }^{13}$ Eine Ausdehnung des Begriffs auf ethnische, religiöse und sprachliche Minderheiten ist somit ausgeschlossen. Angehörige ethnischer, religiöser und sprachlicher Minderheiten genießen im Rahmen des Art. 14 EMRK daher lediglich als Individuum mit den Merkmalen „Rasse“, „Sprache“ und „Religion“ Schutz vor Diskriminierung.

Von Art. 14 EMRK abgesehen gewährleistet die EMRK nur einen „unspezifischen Minderheitenschutz" ${ }^{14}$ : Der Schutz, der den Angehörigen nationaler Minderheiten zuteilwird,knüpft nämlich geradenichtdaran an, dass die Betroffenen Angehörige einer nationalen Minderheit sind, sondern er gründet sich auf den individualrechtlichen Ansatz der EMRK, der den Schutz des Individuums sicherstellen will. ${ }^{15}$ Der von diesen individualrechtlichen Garantien ausgehende Rechtsschutz kommt aber auch den Minderheitenangehörigen - als sich dem Kollektiv „Minderheit“"zugehörig fühlenden Individuen - zugute, da sich diese wie alle anderen Personen im Geltungsbereich der EMRK als Individuum auf die ihnen in der EMRK zugesprochenen Rechte berufen können. ${ }^{16}$ Zusammenfassend wird Minderheitenschutz in der EMRK somit individuell für jeden Minderheitenangehörigen „durch eine Verbindung einer einzelnen Freiheitsgewährleistung mit dem allgemeinen Diskriminierungsverbot des Art. $14[\mathrm{EMRK}]{ }^{“ 17}$ sichergestellt.

\subsection{Zusatzprotokolle zur Europäischen Menschenrechtskonvention (EMRK-Protokolle)}

Zur EMRK existiert eine Vielzahl von Zusatzprotokollen. Ebenso wie die EMRK enthalten die bislang ratifizierten und in Kraft getretenen Zusatzprotokolle jedoch keine speziellen Minderheitenrechte, sondern lediglich Jedermann-Rechte, auf die sich Angehörige nationaler Minderheiten ebenso wie alle anderen Einzelpersonen im Geltungsbereich der EMRK berufen können.

Erwähnenswert sind an dieser Stelle vor allem das 11. Protokoll und das 12. Protokoll zur EMRK. Im 11. Protokoll zur EMRK vom 1.11.1998 ${ }^{18}$ wurde die

13 Vgl. EGMR-Urteil vom 23.7.1968, abgedruckt bei: Berger, V. Rechtsprechung des Europäischen Gerichtshofs für Menschenrechte. Köln, 1987: S. 15 ff.

14 Hillgruber, C.; Jestaedt, M. Die Europäische Menschenrechtskonvention und der Schutz nationaler Minderheiten. Bonn, 1993: S. 89.

15 Vgl. Hillgruber, C. Minderheitenschutz im Rahmen der Europäischen Menschenrechtskonvention - Stand und Entwicklung, in: Blumenwitz, D.; Gornig, G. H. (Hrsg.) Minderheitenund Volksgruppenrecht in Theorie und Praxis. Köln, 1993: S. 39; Hillgruber, C.; Jestaedt, M.: S. 90.

16 Vgl. eingehend zum minderheitenschützenden Charakter der Individualrechte der EMRK: Hillgruber, C.: S. $40 \mathrm{ff}$.

17 Klugmann, M. Europäische Menschenrechtskonvention und antiterroristische Maßnahmen. Frankfurt a. M., 2002: S. 43.

Text siehe: BGBl. 1995 II: S. 579-591. 
Einrichtung eines neuen ständigen Europäischen Gerichtshofs für Menschenrechte (EGMR) beschlossen, der die Europäische Menschenrechtskommission und den bisherigen Europäischen Gerichtshof für Menschenrechte ersetzte. Vor diesem Gerichtshof kann jeder Bürger - aber auch eine Personengruppe - der Unterzeichnerstaaten, der eine Verletzung seiner ihm nach der EMRK zustehenden Rechte und Freiheiten durch einen Vertragsstaat geltend macht, nach Erschöpfung aller innerstaatlichen Rechtsbehelfe eine Menschenrechtsbeschwerde beim EGMR einlegen. ${ }^{19}$ Somit steht mittlerweile etwa 800 Millionen Einwohnern der 47 Vertragsstaaten der EMRK das Individualbeschwerderecht vor dem EGMR zu. ${ }^{20}$ Gegenstand der Menschenrechtsbeschwerde kann hoheitliches Handeln oder Unterlassen der Gerichtsbarkeit, der Verwaltung und unter Umständen auch des Gesetzgebers sein.

ZurVollziehungderUrteiledesEGMRgibteskeinenDurchsetzungsmechanismus. Vielmehr kann das Ministerkomitee des Europarates die Vollziehung der Urteile lediglich überwachen und mit diplomatischen Mitteln durchzusetzen versuchen. Zwangsmaßnahmen zur Vollstreckung eines Urteils gegen den Willen des betreffenden Staates sind nicht vorgesehen. ${ }^{21}$ Bei endgültiger Weigerung des betreffenden Staates besteht letztlich nur noch die Möglichkeit des Ausschlusses aus dem Europarat. $^{22}$

Zentrale Norm des 12. Protokolls zur EMRK vom 4.11.2000 23 ist der in dessen Art. 1 enthaltene allgemeine Gleichheitssatz. Dieser enthält in Art. 1 I ein Diskriminierungsverbot hinsichtlich der auch in Art. 14 EMRK genannten Differenzierungskriterien. Das Diskriminierungsverbot des Art. 1 des 12. Protokolls geht aber in seinem Schutzbereich weiter als Art. 14 EMRK und ergänzt diesen dahingehend, dass es sich nicht nur auf die in der EMRK garantierten Rechte bezieht, sondern auch auf solche, die durch die Rechtsordnungen der einzelnen Mitgliedsstaaten gewährleistet werden. Bei sonst gleichem Wortlaut bezieht sich das Diskriminierungsverbot des Art. 1 I des 12. Protokolls zur EMRK nämlich auf den „Genuss eines jeden gesetzlich niedergelegten Rechtes “24, wohingegen sich das Diskriminierungsverbot des Art. 14 EMRK auf den „Genuss der in dieser Konvention anerkannten Rechte

19 Vgl. Art. 34, 35 EMRK.

20 Vgl. Berger, S. Europarat - Ein Auslaufmodell. Hamburg, 2008: S. 4; Peukert, W. Neueste Entwicklungen des Menschenrechts- und Minderheitenschutzes - Übersicht über die Rechtsprechung des Europäischen Gerichtshofs für Menschenrechte, in: Blumenwitz, D.; Gornig, G. H.; Murswiek, D. (Hrsg.) Fortschritte im Beitrittsprozess der Staaten Ostmittel-, Ost- und Südosteuropas zur Europäischen Union. Köln, 1999: S. 37.

21 Vgl. Art. 46 EMRK.

22 Vgl. Peukert, W.: S. 40.

23 European Treaty Series No. 177.

24 Art. 1 I des 12. Zusatzprotokolls zur EMRK. 
und Freiheiten " ${ }^{25}$ beschränkt. So werden zum einen auch Ungleichbehandlungen bzgl. solcher Rechte erfasst, die nicht in der EMRK enthalten sind, zum anderen ist aufgrund fehlender Akzessorietät die Betroffenheit eines Rechts der EMRK nicht mehr Voraussetzung für die Anwendbarkeit des Diskriminierungsverbots, sondern die Verletzung jeglicher (nationaler oder internationaler) Rechte. ${ }^{26}$

\subsection{Europäische Charta für regionale und Minderheitensprachen von 1992}

Ziel der Europäischen Charta für regionale und Minderheitensprachen vom 5.11.1992 27 ist die Förderung des sprachlichen Erbes zur Bewahrung des kulturellen Reichtums Europas. ${ }^{28}$ Die Charta ist das bislang einzige offizielle internationale Dokument, das sich - zumindest am Rande - mit dem Minderheitenbegriff beschäftigt. Sie liefert zwar keine Definition der „sprachlichen Minderheit“, dafür aber der „minderheitlichen Sprache“. Der Begriff "Regional- oder Minderheitensprache“ wird in Art. 1 a) definiert als Sprache, die herkömmlicherweise in einem bestimmten Gebiet eines Staates von Angehörigen dieses Staates, die eine im Verhältnis zur übrigen Bevölkerung zahlenmäßig kleinere Gruppe bilden, gebraucht wird und die sich von der/den Amtssprache(n) des Staates unterscheidet, ohne deren Dialekt oder eine Sprache von Zuwanderern zu sein. ${ }^{29}$

Die Charta konstituiert zwar weder kollektive noch individuelle Rechte von Minderheitenangehörigen. Aber auf dem für Stiftung und Erhalt der ethnischen und sozialen Identität einer Minderheit existentiell wichtigen Gebiet der eigenen Sprache ${ }^{30}$ schreibt die Charta zumindest gemeinsame Ziele und Verpflichtungen zur Förderung des Gebrauchs der Minderheitensprachen im Schulwesen, in den Medien, im Rechts- und Verwaltungswesen sowie im Wirtschafts-, Sozial- und

Art. 14 EMRK.

Vgl. Grabenwarter, C. Europäische Menschenrechtskonvention. München, 2003: S. 439 f.; Peters, A. Einführung in die Europäische Menschenrechtskonvention. München, 2003: S. 229 f. European Treaty Series No. 148 bzw. BGBl. 1998 II: S. 1314 ff.

Vgl. Art. 7 I a) der Europäischen Charta für regionale und Minderheitensprachen vom 5.11.1992.

Art. 1 a) der Europäischen Charta für regionale und Minderheitensprachen vom 5.11.1992.

Vgl. zur identitätsstiftenden und erhaltenden Bedeutung der Minderheitensprache: BottBodenhausen, K. Die Bedeutung der Kultur für die Wahrung der Identität der Volksgruppel Minderheit, in: Blumenwitz, D.; Gornig, G. H. (Hrsg.) Minderheiten- und Volksgruppenrechte in Theorie und Praxis. Köln, 1993: S. 101; Koplin, B. Nationale und ethnische Minderheiten im Verfassungsrecht der osteuropäischen Staaten. Berlin: Verlag Arno Spitz, 1995: S. 264; Oeter, S. Minderheiten zwischen Segregation, Integration und Assimilation - Zur Entstehung und Entwicklung des Modells der Kulturautonomie, in: Blumenwitz, D.; Gornig, G. H.; Murswiek, D. (Hrsg.) Ein Jahrhundert Minderheiten- und Volksgruppenrechte. Köln, 2001: S. 66. 
Kulturbereich fest. ${ }^{31}$ Die Charta für regionale und Minderheitensprachen stärkt somit die Minderheitensprachen als Bollwerk gegen den in Form der Mehrheitssprache ununterbrochen auf die Minderheit einwirkenden Assimilierungsdruck.

Mechanismen zur Ahndung von Verletzungen der Pflichten der Vertragsstaaten aus den Art. 8-14 der Charta sind nicht vorgesehen. Allein die Pflicht der Vertragsstaaten zur regelmäßigen Veröffentlichung von Berichten über den Stand der Verwirklichung der im Teil III der Charta festgeschriebenen Verpflichtungen ${ }^{32}$ könnte geringen politischen Druck zur Einhaltung der vertraglich übernommenen Verpflichtungen ausüben. Sanktionsmöglichkeiten bei Weigerung zur (korrekten) Abfassung des Berichts fehlen jedoch.

\subsection{Rahmenübereinkommen zum Schutz nationaler Minderheiten von 1995}

Das Rahmenübereinkommen zum Schutz nationaler Minderheiten vom 1.2.1995 ${ }^{33}$, das am 1.2.1998 in Kraft getreten ist, ist der erste multilaterale völkerrechtliche Vertrag, der ausschließlich den Schutz von Angehörigen nationaler Minderheiten zum Inhalt hat. ${ }^{34}$ Es hat die Funktion, einen über die Grenzen des Europarates hinausgehenden minderheitenschutzrechtlichen Mindeststandard festzulegen, der unter strenger Einhaltung des Rechtsstaatsprinzips bei gleichzeitiger Respektierung der territorialen Integrität und nationalen Souveränität der Mitgliedsstaaten $\mathrm{zu}$ erfolgen hat. ${ }^{35}$

Im Gegensatz zum dem Rahmenabkommen zugrundeliegenden Entwurf der Konvention für den Schutz der Minderheiten vom 8.2.1991 ${ }^{36}$ enthält das Rahmenabkommen selbst keine Definition des Begriffs „nationale Minderheit“. Jedoch werden in Art. 5 I vier Elemente genannt, die für die eigenständige Identität einer nationalen Minderheit als essentiell angesehen werden: Religion, Sprache, Tradition und kulturelles Erbe. ${ }^{37}$

Das Rahmenabkommen gewährt keine kollektiven Minderheitenrechte, sondern stellt klar, dass es ausschließlich den Schutz von Angehörigen nationaler

31 Art. 7-14 der Europäischen Charta für regionale und Minderheitensprachen vom 5.11.1992.

32 Art. 15 der Europäischen Charta für regionale und Minderheitensprachen vom 5.11.1992.

33 European Treaty Series No. 157 bzw. BGBl. 1997 II: S. 1407 ff.

34 Vgl. Wheatley, S. The Framework Convention for the Protection of Minorities, in: EHRLR 6 (1996): S. 584.

35 Vgl. Präambel des Rahmenübereinkommens zum Schutz nationaler Minderheiten vom 1.2.1995.

36 Vgl. unten unter II. 4.) d) bb) (1). Text siehe: Europäische Kommission für die Demokratie durch das Recht, Europarat, 4.3.1991, CDL (91) 7.

37 Art 5 II des Rahmenübereinkommens zum Schutz nationaler Minderheiten vom 1.2.1995. 
Minderheiten kodifiziert, die ihre Rechte individuell und in Gemeinschaft mit anderen ausüben. ${ }^{38}$

Das Rahmenabkommen enthält auch keine für die Unterzeichnerstaaten unmittelbar rechtlich verpflichtenden Bestimmungen zum Minderheitenschutz, sondern lediglich innerstaatlich nicht unmittelbar anwendbare programmatische Sätze mit Zielvorgaben zum Minderheitenschutz. Unter anderem finden sich im Rahmenabkommen Bestimmungen zum Existenzrecht nationaler Minderheiten, zur Nichtdiskriminierung und Gleichheit vor dem Gesetz, zum individuellen Bekenntnis zur Minderheitenzugehörigkeit, zum Gebrauch der eigenen Sprache und des eigenen Namens, zu zweisprachigen Orts- und Straßenbezeichnungen in den traditionellen Siedlungsgebieten einer Minderheit erheblichen Ausmaßes, zum Recht auf Erziehung und Bildung in der Minderheitensprache, zu kulturellen Rechten sowie zum Recht auf Kontaktaufnahme mit Minderheitenangehörigen in anderen Staaten. ${ }^{39}$ Der weite Ermessensspielraum der Vertragsstaaten beim Ergreifen von Maßnahmen zum Minderheitenschutz und der bewusst fehlende Sanktionsmechanismus überlassen den vertraglich geforderten effektiven Minderheitenschutz weitestgehend dem guten Willen der Unterzeichnerstaaten.

\subsubsection{Definition des Minderheitenbegriffs in nicht verabschiedeten Entwürfen des Europarates ${ }^{40}$}

\subsection{Entwurf der Konvention für den Schutz der Minderheiten von 1991}

Am 4.3.1991 wurde der Entwurf der Konvention für den Schutz der Minderheiten ${ }^{41}$ von der Europäischen Kommission für Demokratie durch Recht (sog. Venice-Commission) der Versammlung des Europarates vorgelegt. Dieser Entwurf definiert in Art. 2 I „Minderheit“ als zahlenmäßig gegenüber der restlichen Bevölkerung des Staates kleinere Gruppe, deren Mitglieder Staatsangehörige des Wohnsitzstaates sind und ethnische, religiöse oder sprachliche Eigenheiten

38 Vgl. Art 3 II des Rahmenübereinkommens zum Schutz nationaler Minderheiten vom 1.2.1995.

39 Vgl. Abschnitt II (Art. 4-19) des Rahmenübereinkommens zum Schutz nationaler Minderheiten vom 1.2.1995.

40 Neben den im Folgenden näher erläuterten Entwürfen gibt es noch einen Entwurf eines Zusatzprotokolls zur EMRK seitens der Europäischen Akademie Bozen aus dem Jahre 1998. Auf diesen wird jedoch nicht näher eingegangen, da es sich bei der Europäischen Akademie Bozen um eine nichtstaatliche Organisation handelt. Text siehe: Europäische Akademie Bozen. Package for Europe. Measures for Human Rights, Minority Protection, Cultural Diversity and Economic and Social Cohesion. Bozen, 1998).

41 Text siehe: Europäische Kommission für die Demokratie durch das Recht, Europarat, 4.3.1991, CDL (91) 7 oder Human Rights Law Journal 12 (1991): S. 269 ff. 
besitzen, die sie vom Rest der Bevölkerung unterscheiden, und die den Willen haben, ihre Kultur, ihre Traditionen, ihre Religion oder Sprache zu bewahren. ${ }^{42}$ Dieser Entwurf wurde von der Versammlung des Europarates abgelehnt, die sich für ein Zusatzprotokoll zur EMRK und gegen ein gesondertes Abkommen aussprach. Gründe dafür waren die Vermeidung von Überschneidungen mit der EMRK und die Nutzung des bereits existierenden Instrumentariums der EMRK. ${ }^{43}$ Später führte der Konventionsentwurf aber zum Rahmenübereinkommen zum Schutz nationaler Minderheiten vom 1.2.1995 ${ }^{44}$, in dem die Minderheitendefinition jedoch nicht mit aufgenommen wurde.

\subsubsection{2. Österreichischer Entwurf eines Zusatzprotokolls zur EMRK von 1991}

Am 26.11.1991 legte die österreichische Delegation dem Ministerrat einen Entwurf eines Zusatzprotokolls zur Europäischen Menschenrechtskonvention zum Schutz von Volksgruppen ${ }^{45}$ vor. Dieser enthält in Art. 1 eine Definition des Begriffs „Volksgruppe ${ }^{\text {"46. }}$

„Unter dem Begriff „Volksgruppe“ ist eine Gruppe von Bürgern eines Staates zu verstehen, die (a) im Hoheitsgebiet eines Staates traditionell ansässig ist, (b) sich gegenüber der Mehrheitsbevölkerung des Staates oder einer Region eines Staates zahlenmäßig in der Minderheit befindet, (c) gemeinsame ethnische oder sprachliche Merkmale aufweist, die sie von der übrigen Bevölkerung unterscheiden, und (d) über eine eigene kulturelle Identität verfügt. " ${ }^{47}$

Neben dieser Minderheitendefinition enthält der Entwurf noch einen umfassenden Rechtskatalog zum Minderheitenschutz. ${ }^{48}$

42 Art. 2 I des Entwurfs einer Minderheitenschutzkonvention der Europäischen Kommission für Demokratie durch Recht vom 4.3.1991.

43 Vgl. Streinz, R.: S. 23.

44 Vgl. oben unter II. 4.) d) aa) (4).

$45 \mathrm{CDDH}$ (91) 46. Abgedruckt in: Hofmann, R. Minderheitenschutz in Europa. Köln, 1995: S. 252-256.

46 Der Begriff der „Volksgruppe“ ist hier mit dem der „nationalen Minderheit“ gleichzusetzen. Die Verwendung des Begriffs „Volksgruppe“ anstelle des der „nationalen Minderheit“ dürfte auf die Haltung einflussreicher Mitglieder des Europarates zurückzuführen sein, die keine „nationalen“"Minderheiten anerkennen. Vgl. Hofmann, R.: S. 43.

47 Art. 1 des österreichischen Entwurfs eines Zusatzprotokolls zur Europäischen Menschenrechtskonvention zum Schutz von Volksgruppen vom 26.11.1991.

48 Vgl. Art. 3-8 des österreichischen Entwurfs eines Zusatzprotokolls zur Europäischen Menschenrechtskonvention zum Schutz von Volksgruppen vom 26.11.1991. 


\subsection{Parlamentarischer Entwurf eines Zusatzprotokolls zur EMRK von 1993}

Der Entwurf eines Zusatzprotokolls zur EMRK vom 1.2.1993 ${ }^{49}$ wurde dem gesetzgebenden Ministerrat des Europarates zwar von der parlamentarischen Versammlung des Europarates mit Dreiviertelmehrheit zur Annahme empfohlen (Empfehlung 1201), der die Annahme des Zusatzprotokolls aber am 7.10.1993 in Wien ablehnte. ${ }^{50}$ Der Entwurf enthielt eine im Kern der Definition Capotortis ähnelnde, aber detailliertere Minderheitendefinition:

Nationale Minderheiten wurden dort definiert als eine Gruppe von Personen in einem Staat, die (a) im Hoheitsgebiet dieses Staates ansässig und dessen Staatsbürger sind, (b) langjährige, feste und dauerhafte Verbindungen zu diesem Staat aufrechterhalten, (c) besondere ethnische, kulturelle, religiöse oder sprachliche Merkmale aufweisen, (d) ausreichend repräsentativ sind, obwohl ihre Zahl geringer ist als die der übrigen Bevölkerung dieses Staates oder einer Region dieses Staates, (e) von dem Wunsch beseelt sind, die für ihre Identität charakteristischen Merkmale, insbesondere ihre Kultur, ihre Traditionen, ihre Religion oder ihre Sprache gemeinsam zu erhalten. ${ }^{51}$

\subsection{6. $\mathrm{EG} / \mathrm{EU}$}

Der Minderheitenschutz ist in der EG/EU in erster Linie ein Aspekt der Gemeinsamen Außen- und Sicherheitspolitik (GASP). Auch in Resolutionen des Europäischen Parlaments fand eine Auseinandersetzung mit dem Minderheitenproblem statt.

\subsubsection{Bestimmungen zum Minderheitenschutz in EG/EU auf parlamentarischer Ebene}

Zwar beschäftigten sich zahlreiche Dokumente des Europäischen Parlaments (EP) mit dem Minderheitenproblem, aber keines dieser Dokumente enthielt eine Definition des Begriffs „Minderheit“. Zu nennen sind an dieser Stelle z. B. die Resolution des EP über eine Charta der Rechte der ethnischen Minderheiten vom 16.10.1981 $1^{52}$, die Resolution des EP über Maßnahmen zugunsten der

\footnotetext{
49 Bundesrats-Drs. 12/4572: S. 12 ff. Abgedruckt in: EuGRZ 1993: S. 151 ff.

50 Vgl. Wiener Erklärung vom 7.10.1993, in: Bulletin der Bundesregierung Nr. 91/1993: S. $1021 \mathrm{ff}$.

51 Zitiert nach Steiner, L.: S. 37.

52 Resolution vom 15. und 16.10.1981 nach einer Debatte über die Berichte 1-371/79 und 1965/80. Text siehe: Abl. 1981 Nr. C 287: S. 106 f.
} 
minderheitlichen Sprachen und Kulturen vom 11.2.1983 ${ }^{53}$, die Resolution des EP über die Sprachen und Kulturen der regionalen und ethnischen Minderheiten der Europäischen Gemeinschaft vom 30.10.198754, die Resolution des EP vom 28.1.1991 sowie die Resolution des EP über die linguistischen und kulturellen Minderheiten in der EU vom 9.2.199455.

Ein Vorschlag des Europäischen Parlaments, die Anerkennung und den Schutz von Minderheiten in den EU-Vertrag aufzunehmen, wurde nicht weiter diskutiert, so dass der EU-Vertrag nach wie vor keine Bestimmungen zu Minderheiten enthält. ${ }^{56}$

1984 legte der Abgeordnete Goppel einen Berichtsentwurf zu den Rechten der Volksgruppen und Minderheiten vor. Nach einem entsprechenden Entschließungsantrag von 42 Abgeordneten wurde der Entschließungsentwurf an den Ausschuss für Recht und Bürgerrechte überwiesen. ${ }^{57}$ Dessen Berichterstatter Graf Stauffenberg legte seinen Berichtsentwurf für eine Charta der Volksgruppenrechte in den Staaten der Europäischen Gemeinschaft 1988 vor. Dieser gelangte jedoch nicht bis zur Endabstimmung im Plenum. ${ }^{58}$ Der Entwurf Graf Stauffenbergs für eine Charta der Volksgruppenrechte enthält als einziges EG/EU-Dokument eine - bislang allerdings von keiner EU-Institution offiziell anerkannte - Minderheitendefinition ${ }^{59}$ : „Als Volksgruppe im Sinne dieser Charta gilt $[\mathrm{a}]^{60}$ die Gesamtheit all jener Staatsbürger eines Mitgliedsstaates der Europäischen Gemeinschaft, [b] die im Staatsgebiet traditionell in der Folge vieler Generationen ansässig sind, [c] gemeinsame ethnische, religiöse und/oder sprachliche Merkmale aufweisen, die sie von anderen Bevölkerungsteilen unterscheiden, [d] gemeinsam über eine eigene kulturelle Identität verfügen und [e] sich innerhalb der Gesamtbevölkerung des Staates in Minderheit befinden." ${ }^{\circ 1}$

53 Resolution 89/C153/02. Text siehe: EuGRZ 1983: S. $143 \mathrm{ff}$.

54 Text siehe: EuGRZ 1987: S. 607 f.

55 Resolution A3-0042/94.

56 Vgl. Witte, B. de. The European Community and its Minorities, in: Brölmann, C.; Lefeber, R.; Zieck, M. (Hrsg.) Peoples and Minorities in International Law. Dordrecht, 1993: S. 179.

57 Text siehe: Europa Ethnica 1984: S. 233 f.

58 Entwurf eines Berichts über eine Charta der Volksgruppenrechte in den Staaten der Europäischen Gemeinschaften vom 17.3.1988, in: Europa als Auftrag, Schriftenreihe der EVPFraktion im Europäischen Parlament, Heft 3 (1991): S. 3 ff.

59 Der in Graf Stauffenbergs Definition verwendete Begriff der „Volksgruppe“ ist mit dem der "nationalen Minderheit“ gleichzusetzen. Die Verwendung des Begriffs „Volksgruppe“ anstelle des der „nationalen Minderheit“ dürfte auf die Haltung einflussreicher Mitglieder des Europarates zurückzuführen sein, die keine „nationalen“ Minderheiten anerkennen.

60 Die in [ ] gesetzten Nummerierungszeichen finden sich nicht im Originaltext, sondern sind Ergänzungen des Autors zur Hervorhebung der einzelnen Kriterien einer Minderheit.

61 Entwurf eines Berichts über eine Charta der Volksgruppenrechte in den Staaten der Europäischen Gemeinschaften vom 17.3.1988, in: Europa als Auftrag, Schriftenreihe der EVPFraktion im Europäischen Parlament, Heft 3 (1991): S. 3. 
Eine ähnliche Definition wurde im Bericht des neuen Berichterstatters Alber vom 14.5.1993 vorgeschlagen. ${ }^{62}$

In einer Resolution zum Minderheitenschutz und zur Anti-Diskriminierungspolitik empfahl das Europaparlament am 8.6.2005 die Übernahme der Minderheitendefinition in der Empfehlung 1201 des Europarates vom 1.2.1993. Dabei beklagte das Europaparlament das Fehlen eines allgemein anerkannten Minderheitenbegriffs auch in der EU. ${ }^{63}$

\subsubsection{Bestimmungen zum Minderheitenschutz in EG/EU auf politischer Ebene}

Auf politischer Ebene wurden verschiedene Erklärungen zur Minderheitenfrage abgegeben, die jedoch allesamt ebenfalls keine Definition des Begriffs „Minderheit“ enthielten. Herausragende Bedeutung hat hier die Erklärung der EG-Außenminister zu den „Richtlinien für die Anerkennung neuer Staaten in Osteuropa und der Sowjetunion"vom Dezember 1991. Darin bekräftigen die (damals noch) EG und ihre Mitgliedsstaaten, dass sie sich der UN-Charta, den Prinzipien der Schlussakte von Helsinki und der Charta von Paris, insbesondere im Hinblick auf Rechtsstaatlichkeit, Demokratie und Menschenwürde, verpflichtet fühlen. Sie fordern vor der Anerkennung neuer Staaten, dass diese die Garantien für die Rechte ethnischer und nationaler Gruppen und Minderheiten im Einklang mit den im Rahmen der KSZE eingegangenen Verpflichtungen respektieren und die Unverletzlichkeit aller Grenzen, die nur auf friedlichem Weg und einvernehmlich geändert werden dürfen, achten. ${ }^{64}$ Damit wurde die Anerkennung von Minderheitenrechten neben der Achtung der (bestehenden) territorialen Integrität erstmals zur konstitutiven Voraussetzung für die Anerkennung eines Staates durch die europäische Staatengemeinschaft.

\subsubsection{EU-Richtlinien zur Gleichbehandlung und Antidiskriminierung}

AucheinigeRichtlinienderEUenthaltenBestimmungenzumMinderheitenschutz, ohne allerdings den Begriff „Minderheit“ in ihren Schutzbestimmungen ausdrücklich

62 Vgl. Doc. PE, 204.838.

63 Entschließung des Europäischen Parlaments zum Schutz von Minderheiten und den Maßnahmen

gegen Diskriminierung in einem erweiterten Europa (2005/2008(INI)), in: ABl. C124 E, S. 407, Paragraph 7.

64 Vgl. Richtlinien für die Anerkennung neuer Staaten in Osteuropa und in der Sowjetunion vom 16.12.1991, in: Europa-Archiv, Folge 2/1992, D 120. 
zu erwähnen. So verbietet die Antidiskriminierungsrichtlinie vom 29.6.2000 ${ }^{65}$ unmittelbare oder mittelbare Diskriminierung aus Gründen der Rasse oder der ethnischen Herkunft bei den Einstellungsbedingungen und Auswahlkriterien für einen Arbeitsplatz, beim Zugang zu Berufsberatung, Berufsausbildung und -weiterbildung, bei Beschäftigungs- und Arbeitsbedingungen, bei der Mitgliedschaft in Arbeitgeber- oder Arbeitnehmerorganisationen, bei Sozialschutz, Gesundheitsdiensten, Bildung sowie dem Zugang zu Gütern und Dienstleistungen einschließlich Wohnraum. Allerdings bezeichnet sich die Antirassismusrichtlinie selbst als Teil eines „Bündel[s] aufeinander abgestimmter Maßnahmen [...], die darauf abstellen, Diskriminierungen bestimmter gesellschaftlicher Gruppen, wie ethnischer Minderheiten, zu bekämpfen" ${ }^{\text {66 }}$.

Die Gleichbehandlungsrahmenrichtlinie vom 27.11.2000 ${ }^{67}$ bildet einen Eckpfeiler des Minderheitenschutzes der EU. ${ }^{68}$ Sie untersagt die Diskriminierung in Beschäftigung und Beruf aufgrund von Religion, Weltanschauung, Behinderung, Alter oder sexueller Ausrichtung. Der Begriff „Minderheit“ wird hier jedoch an keiner Stelle erwähnt.

Somit gründet sich der Schutz dieser Richtlinien, der auch Minderheitenangehörigen zuteilwird, gerade nicht auf deren Zugehörigkeit zu einer Minderheit, sondern auf den individualrechtlichen Ansatz der jeweiligen Richtlinie, die den Schutz des Individuums bezweckt.

\subsubsection{Charta der Grundrechte der Europäischen Union von 2000}

In der Charta der Grundrechte der Europäischen Union vom 7.12.2000 ${ }^{69}$ finden nationale Minderheiten Erwähnung, ohne dass jedoch definiert wird, welche Personengruppen unter den Begriff „nationale Minderheit“ subsumiert werden können.

Art. 22 der Charta enthält ein klares Bekenntnis der EU zur kulturellen, religiösen und sprachlichen Vielfalt in Europa. ${ }^{70}$ In der Formulierung ähnlich, nur etwas differenzierter in den Unterscheidungskriterien als in Art. 14 EMRK, werden in Verwirklichung des Art. 13 des durch den Amsterdamer Vertrag modifizierten EUVertrages nationale Minderheiten im Diskriminierungsverbot des Art. 21 der Charta geschützt:

ABl. L 180/22 vom 19.7.2000.

Erwägungsgrund 8 der Richtlinie 2000/43/EG.

ABl. L 303/16 vom 2.12.2000.

Vgl. Schwellnus, G. Anti-discrimination legislation, in: Rechel, B. (Ed.) Minority Rights in Central and Eastern Europe. London, 2009: S. 33.

ABl., 2000 C 364: S. 1 ff.

ABl., 2000 C 364: S. 13. 
„Diskriminierungen, insbesondere wegen des Geschlechts, der Rasse, der Hautfarbe, der ethnischen oder sozialen Herkunft, der genetischen Merkmale, der Sprache, der Religion oder der Weltanschauung, der politischen oder sonstigen Anschauung, der Zugehörigkeit zu einer nationalen Minderheit ${ }^{71}$, des Vermögens, der Geburt, einer Behinderung, des Alters oder der sexuellen Ausrichtung, sind verboten. ${ }^{\text {"72 }}$

Ebenso wie Art. 14 EMRK gewährt Art. 21 der Charta lediglich „unspezifischen Minderheitenschutz ${ }^{\text {“73. }}$. Denn der Schutz, der den Angehörigen nationaler Minderheiten zuteilwird, knüpft gerade nicht daran an, dass die Betroffenen Angehörige einer nationalen Minderheit sind, sondern er gründet sich auf den individualrechtlichen Ansatz der Charta, der den Schutz des Individuums sicherstellen will. Neben den ausdrücklich genannten Angehörigen nationaler Minderheiten genießt aber auch jeder Angehörige ethnischer, religiöser und sprachlicher Minderheiten als Individuum mit den Merkmalen „Rasse“, „Sprache“ und „Religion“ im Rahmen des Art. 21 Schutz vor Diskriminierung.

\subsubsection{Vertrag über die Arbeitsweise der Europäischen Union von 2007}

Mit dem am 1.12.2009 in Kraft getretenen Vertrag über die Arbeitsweise der Europäischen Union (AEUV) $)^{74}$ - Vertrag von Lissabon - fanden Minderheiten erstmals Erwähnung im Primärrecht der EU. Nunmehr bezieht sich Art. 2 EUVertrags (EUV) ausdrücklich darauf, dass die „Werte, auf die sich die Union gründet, [...] die Achtung der Menschenwürde, Freiheit, Demokratie, Gleichheit, Rechtsstaatlichkeit und die Wahrung der Menschenrechte einschließlich der Rechte der Personen, die Minderheiten angehören" sind. Der Begriff der Minderheit wird jedoch weder im Vertrag von Lissabon noch in der konsolidierten Fassung des EUVertrages näher umschrieben oder definiert. Das heißt zugleich aber auch, dass der Minderheitenbegriff im Primärrecht der EU nicht auf bestimmte Arten von Minderheiten beschränkt wird, sondern eher weit gefasst ist.

\subsubsection{Rahmenbeschluss zur Bekämpfung von Rassismus und Fremdenfeindlichkeit von 2008}

Im Rahmenbeschluss 2008/913/JI des Rates vom 28.11.2008 zur strafrechtlichen Bekämpfung bestimmter Formen und Ausdrucksweisen von Rassismus und

71 Textliche Hervorhebung des Autors.

72 ABl., 2000 C 364: S. 13.

73 Hillgruber, C.; Jestaedt, M.: S. 89.

74 ABl. 2007 C 306/1. 
Fremdenfeindlichkeit ${ }^{75}$ werden Minderheiten und Angehörige einer Minderheit unter besonderen strafrechtlichen Schutz gestellt. Art. 1 des Rahmenbeschlusses schützt „eine nach den Kriterien der Rasse, Hautfarbe, Religion, Abstammung oder nationaler oder ethnischer Herkunft definierte Gruppe von Personen oder [...] ein Mitglied einer solchen Gruppe "vor rassistischen oder fremdenfeindlichen Handlungen. Vom Tatbestandsmerkmal „nach nationaler oder ethnischer Herkunft definierte Gruppe oder ein Mitglied einer solchen Gruppe“ sind auch Minderheiten und deren Angehörige umfasst. Der Begriff der Minderheit wird hingegen im Rahmenbeschluss weder erwähnt noch näher definiert.

\section{Gegenwärtiger Minderheitenbegriff der EU}

\subsection{Keine allgemein anerkannte Minderheitendefinition}

Wie die obigen Ausführungen gezeigt haben, existiert in der EU keine allgemein anerkannte und verbindliche Minderheitendefinition. Auch aus Dokumenten der UN, der UNESCO, der KSZE/OSZE und des Europarates lässt sich keine Definition des Begriffs „Minderheit“ entnehmen. Dies ist darauf zurückzuführen, dass ein Konsens aufgrund der unterschiedlichen Interessenlagen der einzelnen Staaten nicht $\mathrm{zu}$ erreichen war. Während einige Staaten innerstaatlich weitreichenden Minderheitenschutz gewährleisten und auch auf völkerrechtlicher Ebene dafür eintreten ${ }^{76}$ gibt es andere Staaten - so z. B. Frankreich, und Griechenland $-{ }^{77}$, die traditionell aufgrund ihrer nationalen Staatsauffassung und zum Teil auch aufgrund von Furcht vor Bedrohung ihrer territorialen Integrität die Existenz von Minderheiten auf ihrem eigenen Staatsgebiet ganz oder zum Teil leugnen und sich demzufolge einer allgemein verbindlichen Minderheitendefinition widersetzen.

Insoweit ist die Wissenschaft mit ihren Erkenntnissen und Definitionsansätzen des Minderheitenbegriffs der politischen Machbarkeit weit voraus. Es herrscht nach wie vor eine große Diskrepanz zwischen rechtstheoretischer Perfektion und politischer Durchsetzbarkeit. Im Bereich des Minderheitenschutzes sind immer noch die Minderheitenschutzvorschriften der nationalen Rechtsordnungen bestimmend. ${ }^{78}$ Dennoch haben sich im politischen Prozess und in den Instanzen der EU trotz unterschiedlicher Auffassungen und Interessen der Mitgliedsstaaten einige Kriterien zur Bestimmung des Minderheitenbegriffs herauskristallisiert, die einem

ABl. L 328/55.

So z. B. Österreich mit seinem Entwurf eines Zusatzprotokolls zur EMRK vom 26.11.1991. Vgl. zum Minderheitenschutz in den EU-Mitgliedsstaaten: Streinz, R.: S. 26 sowie ausführlich: Hofmann, R.: S. $66 \mathrm{ff}$.

Vgl. Hofmann, R.: S. 34.

Vgl. Hofmann, R.: S. $64 \mathrm{f}$. 
gemeinsamen europäischen Minderheitenverständnis zugrunde gelegt werden können.

\subsection{Eigenschaften einer Minderheit nach europäischem Verständnis}

Hauptgarant eines effektiven Minderheitenschutzes in Europa sind die Beitrittsverhandlungen zur EU. In den sog. Kopenhagener Kriterien, welche die Beitrittsvoraussetzungen zur EU normieren, ist der Minderheitenschutz eine der zentralen Voraussetzungen, die ein Land erfüllen muss, um Mitglied in der EU zu werden. Somit konnten die vornehmlich osteuropäischen Staaten seit dem Jahr 2004 der EU auch nur beitreten, nachdem sie innerstaatlich einen ausreichenden Minderheitenschutz sichergestellt hatten. ${ }^{79}$

Zur Bestimmung der zu schützenden Minderheiten haben sich seitens der EU einige regelmäßig aufgegriffene Merkmale herausgebildet, die - trotz fehlender Kodifikation - als konstante und konstituierende Kriterien einer europäischen Minderheitendefinition angesehen werden können. Dabei lassen sich objektive und subjektive Kriterien der Minderheitendefinition unterscheiden, die allesamt kumulativ vorliegen müssen, um eine Personengruppe als Minderheit qualifizieren zu können.

\subsubsection{Objektive Kriterien}

\subsubsection{Zahlenmäßige Unterlegenheit}

Ausgehend vom Wortlaut kann Minderheit nur eine Personengruppe sein, die zahlenmäßig kleiner als der Rest der Bevölkerung des Staates ist. Der numerische Vergleich muss dabei mit der Gesamtzahl der Bevölkerung des betroffenen Staates, d. h. mit der Gesamtzahl aller Staatsangehörigen des Staates einschließlich der Angehörigen der Gruppe, die den Minderheitenstatus beansprucht, vorgenommen werden. ${ }^{80}$

Bei diesem Kriterium ist auch allein die zahlenmäßige Unterlegenheit maßgeblich, so dass materielle Einschränkungen wie Schutzbedürftigkeit, Größenordnung der

79 Vgl. dazu auch: Directorate-General for External Policies. Mainstreaming human and minority rights in the EU enlargement with the Western Balkans. Brussels, 2012: S. 27, 58 ff.; Kurban, D. A Quest for Equality: Minorities in Turkey. Istanbul, 2007: S. 8.

80 Vgl. Ahmed, T. The Impact of EU Law on Minority Rights. Oxford, 2011: S. 23; Gornig, G. H. Die Definition des Minderheitenbegriffs aus historisch-völkischer Sicht: S. 37; Krugmann, M. Das Recht der Minderheiten. Berlin, 2004: S. 63; Topidi, K. EU Law, Minorities and Enlargement. Antwerp, 2010: S. 14. 
zahlenmäßigen Unterlegenheit oder regionale Begrenzung des Bezugsrahmens an dieser Stelle außer Acht bleiben müssen. ${ }^{81}$

\subsubsection{Keine beherrschende Stellung}

Die zahlenmäßig unterlegene Personengruppe darf im Staat keine dominierende Rolle in Form von Alleinentscheidungsbefugnissen haben, da sie in diesem Falle völkerrechtlich nicht schutzwürdig wäre. ${ }^{82}$

Das Abstellen auf die formale Bezugsgröße der Alleinentscheidungsbefugnisse ist erforderlich, da eine materielle Bestimmung der tatsächlichen Einflussmöglichkeiten der Minderheit in jedem Einzelfall unmöglich ist, so dass sich „überwiegender Einfluss" nicht eindeutig feststellen lässt. ${ }^{83}$

\subsubsection{Ethnische, religiöse oder sprachliche Eigenart}

Eine Minderheit im völkerrechtlichen Sinne muss mindestens eine ethnische, religiöse oder sprachliche Eigenart aufweisen, durch die sie sich vom Rest der Bevölkerung unterscheidet. ${ }^{84}$ Als objektives Kriterium muss diese Eigenschaft auch objektives bzw. objektiv erkennbares Kennzeichen der Volksstammzugehörigkeit sein. Damit nicht jeder Einzelne durch die Annahme oder den Wechsel seiner Religion oder durch das Erlernen einer Minderheitensprache Angehöriger einer Minderheit werden kann, bedarf es insbesondere bei religiösen und sprachlichen Eigenarten einer objektivierten, an Tradition anknüpfenden Betrachtung. ${ }^{85}$ Religiöse oder sprachliche Eigenarten können also nur dann zur Begründung der Minderheiteneigenschaft herangezogen werden, wenn sie über eine gewisse Tradition verfügen, d. h. über einen längeren Zeitraum in einem bestimmten Gebiet Bestand hatten. Im Gegensatz zu religiösen und sprachlichen Eigenarten als tatsächlichen Verhaltensweisen ist die

81 Vgl. Krugmann, M.: S. 64 f.; Rehman, J. International Human Rights Law: A Practical Approach. Harlow, 2003: S. 299.

82 Vgl. Ahmed, T.: S. 23; Gornig, G. H. Die Definition des Minderheitenbegriffs aus historischvölkischer Sicht: S. 38; Krugmann, M.: S. 65 ff.; Topidi, K.: S. 14.

83 Vgl. Krugmann, M.: S. 67.

84 Vgl. Capotorti, F. Study on the Rights of Persons belonging to Ethnic, Religious and Linguistic Minorities. New York, 1991: S. 96; Gornig, G. H. Die Definition des Minderheitenbegriffs aus historisch-völkischer Sicht: S. 35; Kizilkan-Kisacik, Z. B. Europeanization of Minority Norms in Turkey. Baden-Baden, 2013: S. 139 f.; Pogge, T. W. 'Group Rights and Ethnicity', in: Shapiro, I.; Kymlicka, W. (Eds.) Ethnicity and Group Rights. New York, 1997: S. 193; Vos, G. A. de. Ethnic Pluralism: Conflict and Accommodation - The Role of Ethnicity in Social History, in: Romanucci-Ross, L.; Vos, G. A. de. Ethnic Identity: Creation, Conflict and Accommodation, $3^{\text {rd }}$ Ed. Walnut Creek, 1995: S. 18.

Vgl. Krugmann, M.: S. 72 f. 
ethnische Abstammung eine historische Tatsache und bedarf daher keines weiteren objektivierenden Maßstabs.

\subsubsection{Staatsangehörigkeit des Aufenthaltsstaates}

Weiteres Kriterium der europäischen Minderheitendefinition ist das Erfordernis der Staatsangehörigkeit im Aufenthaltsstaat. ${ }^{86}$

Das Staatsangehörigkeitskriterium ist zwar - insbesondere im Zusammenhang mit Art. 27 IPBPR - immer wieder kontrovers diskutiert worden. ${ }^{87}$ Auf europäischer Ebene ist die Staatsangehörigkeit im Aufenthaltsstaat aber mittlerweile anerkanntes Kriterium des Minderheitenbegriffs. ${ }^{88}$ So dokumentiert neben dem Berichtsentwurf von Graf Stauffenberg ${ }^{89}$ und den Entwürfen für ein EMRK-Zusatzprotokoll aus den Jahren 1991 und $1993^{90}$ beispielsweise die Empfehlung 1177/1992 des Europarates, dass die Garantie des Schutzes bestimmter kultureller, religiöser oder sprachlicher Eigenschaften an die Staatsangehörigkeit geknüpft ist. ${ }^{91}$ Auf Ausländer findet demnach nicht das Minderheitenrecht, sondern das völkerrechtliche Fremdenrecht und das innerstaatliche Ausländerrecht Anwendung. ${ }^{92}$

Ausnahmen vom Staatsangehörigkeitserfordernis müssen jedoch in den Fällen zugelassen werden, in denen Angehörigen einer faktischen Minderheit die Staatsangehörigkeit seitens des Aufenthaltsstaates willkürlich vorenthalten oder entzogen wird. Umgekehrt ist es für eine Minderheit unschädlich, wenn sich ihr Nichtstaatsangehörige des Aufenthaltsstaates anschließen. ${ }^{93}$

Ohne das Erfordernis der Staatsangehörigkeit im Aufenthaltsstaat könnte kein Staat effektiven Minderheitenschutz gewährleisten, da er sich damit Wanderungs-

86 Vgl. Gornig, G. H. Die Definition des Minderheitenbegriffs aus historisch-völkischer Sicht: S. 40.

87 Vgl. dazu m. w. N.: Ahmed, T.: S. 23 f.; Brunner, G. Nationalitätenprobleme und Minderheitenkonflikte in Osteuropa. Gütersloh, 1996: S. 22 f.; Topidi, K.: S. 14.

88 Vgl. Gornig, G. H. Die Definition des Minderheitenbegriffs aus historisch-völkischer Sicht: S. 40; Oran, B. Lozan'ın „Azınlıkların Korunması “Bölümünü Yeniden Okurken, in: SBF Dergisi, Cilt 49, No. 3-4 (1994): S. 284.

89 Entwurf eines Berichts über eine Charta der Volksgruppenrechte in den Staaten der Europäischen Gemeinschaften vom 17.3.1988, in: Europa als Auftrag, Schriftenreihe der EVPFraktion im Europäischen Parlament, Heft 3 (1991): S. 3 ff.

90 Vgl. Art. 1 des österreichischen Entwurfs eines Zusatzprotokolls zur Europäischen Menschenrechtskonvention zum Schutz von Volksgruppen vom 26.11.1991 (abgedruckt in: Hofmann, R.: S. 252) und parlamentarischer Entwurf eines Zusatzprotokolls zur EMRK vom 1.2.1993 (abgedruckt in: EuGRZ 1993: S. 151 ff.).

91 Vgl. Empfehlung 1177/1992 des Europarates, in: BT-Drs. 12/3018 vom 8.7.1992.

92 Vgl. Brunner, G. Die rechtliche Lage der Minderheiten in Mittel-, Ost- und Südosteuropa, in: OstEuR 1994: S. 164; Gornig, G. H. Die Definition des Minderheitenbegriffs aus historischvölkischer Sicht: S. 41.

93 Vgl. Gornig, G. H. Die Definition des Minderheitenbegriffs aus historisch-völkischer Sicht: S. 41. 
bewegungen von Ausländern ausliefern würde, die bei Erfüllung der übrigen Merkmale der Minderheitendefinition unmittelbaren Minderheitenstatus erhielten und so durch besonderen Minderheitenschutz und Förderung gegenüber den eigenen Staatsangehörigen des Aufenthaltsstaates privilegiert werden würden. ${ }^{94}$ Diese Gefahr bestünde auch, wenn Ausländer, denen kurz nach ihrer Einwanderung die Staatsangehörigkeit verliehen wird, mit der Staatsangehörigkeit ausgestattet nun im neuen Staat unmittelbar eine neue Minderheit bilden könnten. Daher muss in Fällen von eingewanderten Gruppen zur Gewährung eines effektiven Minderheitenschutzes zusätzlich darauf abgestellt werden, ob die betreffende Gruppe bereits über einen längeren Zeitraum mit dem neuen Aufenthaltsstaat verbunden und mittlerweile dort beheimatet ist. ${ }^{95}$ Umstritten dabei ist, wie lange dieser Zeitraum bemessen sein muss. Auf jeden Fall ist dieser „längere Zeitraum“ ab der dritten Generation der Eingewanderten gegeben, da diese damit eindeutig bezeugen, dass sie den Aufenthaltsstaat dauerhaft als neue Heimat ansehen. ${ }^{96}$

\subsubsection{Subjektive Kriterien}

Nach allgemeiner Ansicht führen die oben angeführten objektiv feststellbaren Kriterien allein noch nicht zur Minderheiteneigenschaft. Daneben werden subjektive konstituierende Merkmale gefordert. ${ }^{97}$ Dabei muss allerdings differenziert werden:

94 Vgl. Gornig, G. H. Die Definition des Minderheitenbegriffs aus historisch-völkischer Sicht: S. 39 f.; Krugmann, M.: S. 76.

95 Vgl. Gornig, G. H. Die Definition des Minderheitenbegriffs aus historisch-völkischer Sicht: S. 43; Krugmann, M.: S. 76 ff.; Niewerth, J. Der kollektive und der positive Schutz von Minderheiten und ihre Durchsetzung im Völkerrecht. Berlin, 1996: S. 41 ff.; Pentassuglia, G. Minorities in international law. Strasbourg, 2002: S. 58 f. So beinhaltet die englische Originalfassung des Art. 27 IPBPR auch den Begriff „exist“ (vgl. Nowak, M. UNO-Pakt über bürgerliche und politische Rechte und Fakultativprotokoll, in: Nowak, M. CCPR-Kommentar. Kehl am Rhein, 1989, Rn. 18 zu Art. 27 CCPR).

96 Vgl. dazu auch: Krugmann, M.: S. 77 f.; Veiter, T. Wege zu einem modernen Volksgruppenrecht, in: Aus Politik und Zeitgeschichte, B 18/1975: S. 32.

97 Vgl. u. a. Blumenwitz, D. Minderheiten- und Volksgruppenrecht. Bonn, 1992: S. 121; Çavuşoğlu, N. 2004 AB İlerleme Raporu ve Azınlık kavramı çerçevesinde Kültürel Haklara ilişkin sorunlar, Vortragsmanuskript zum gleichnamigen Vortrag auf der Konferenz der Istanbul Universität vom 8.12.2004 zum Thema „AB’ne Katılım Sürecinde İnsan Hakları“: S. 2; Çavuşoğlu, N. Azınlık Haklarl: Avrupa Standartları ve Türkiye Bir Karşılaştırma, in: Kaboğlu, I. Ö. (Haz.) Azınlık Hakları. Istanbul, 2002: S. 125; Goldman, O. Q. The Need for an Independent International Mechanism to Protect Group Rights: A Case Study of the Kurds, in: 2 Tulsa J. Comp. \& Int'l L. 45 (1994): S. 46 f.; Hofmann, R.: S. 15; Thornberry, P. International Law and the Rights of Minorities. Oxford, 1991: S. 27 f. 


\subsubsection{Zugehörigkeits- oder Solidaritätsgefühl}

Minderheit kann nur eine Personengruppe sein, zu der sich auch Personen bekennen. Subjektives Merkmal zur Feststellung der Minderheiteneigenschaft ist daher das Bekenntnis des Einzelnen zur Zugehörigkeit bzw. Solidarität zu einer Minderheit. ${ }^{98}$

DaeinbloßnachinnengerichtetesZusammengehörigkeits-oderSolidaritätsgefühl als Wille oder Bewusstsein, einer Minderheit anzugehören, für das Gewähren von Minderheitenrechten nicht ausreichen kann, muss es auf irgendeine Art und Weise nach außen in Erscheinung treten. Denn nur, wenn der zum Minderheitenschutz verpflichtete Staat die von ihm zu schützenden Minderheitenangehörigen als solche erkennen kann, kann er diesen auch die ihnen zustehenden Minderheitenrechte zuteilwerden lassen..$^{99}$

Allerdings kommt es nicht bei allen Minderheitenarten auf das Bekenntnis nach außen an. Lediglich bei Angehörigen religiöser und sprachlicher Minderheiten ist ein Bekenntnis nach außen erforderlich, da erst durch dieses Bekenntnis die Zugehörigkeit zur entsprechenden religiösen oder sprachlichen Minderheit registriert werden kann. Bei Angehörigen ethnischer Minderheiten ist ein derartiges Bekenntnis hingegen nicht erforderlich, da diese bereits kraft ihrer Abstammung bzw. Tradition einer Minderheit angehören.

Weil aber niemand aufgrund seines aus der Menschenwürde abgeleiteten Selbstbestimmungsrechts gegen seinen Willen als Angehöriger einer Minderheit behandelt werden darf ${ }^{100}$ und somit nicht ungewollt Adressat von Maßnahmen zum Minderheitenschutz werden darf, wird teilweise vertreten, dass es sich beim subjektiven Merkmal des Zusammengehörigkeits- oder Solidaritätsgefühls „nicht um ein den Minderheitenstatus begründendes Erfordernis, sondern allenfalls um ein Ausschlussmerkmal “101 handelt. Bei dieser Argumentation werden aber zwei verschiedene Ebenen vermischt. Das nach außen kundgetane Bekenntnis zur Zugehörigkeit zu einer Minderheit ist bei religiösen und sprachlichen Minderheiten sehr wohl Definitionskriterium einer Minderheit, da erst durch diese subjektive Komponente die Zugehörigkeit zur entsprechenden religiösen oder sprachlichen Minderheit begründet und manifestiert werden kann. Die Zugehörigkeit zu diesen Minderheitenarten ist willensgesteuert. Durch die Aufgabe der entsprechenden

98 Vgl. Ahmed, T.: S. 24 f.; Gornig, G. H. Die Definition des Minderheitenbegriffs aus historischvölkischer Sicht: S. 44 f.; Oran, B. Küreselleşme ve Azınlıklar, 4. Basım. Ankara, 2001: S. 69; Topidi, K.: S. 14.

99 Vgl. Krugmann, M.: S. 79.

100 So Art. 3 I des Rahmenabkommens zum Schutz nationaler Minderheiten vom 1.2.1995 (abgedruckt in: EuGRZ 1995: S. 269).

101 Krugmann, M.: S. 80. 
religiösen oder sprachlichen Besonderheiten kann die Minderheitenzugehörigkeit auch ohne Weiteres beendet werden. Lediglich die Zugehörigkeit $\mathrm{zu}$ einer ethnischen Minderheit kann nicht durch einen Willensentschluss aufgegeben werden. Diese bei religiösen und sprachlichen Minderheiten bekenntnisabhängige und bei ethnischen Minderheiten de facto bestehende Minderheitenzugehörigkeit ist jedoch streng von der Frage zu trennen, ob der einzelne Minderheitenangehörige die kraft seiner Minderheitenzugehörigkeit bestehenden Minderheitenrechte in Anspruch nehmen oder auf sie verzichten will. Zusammengehörigkeits- oder Solidaritätsgefühl betreffen die Zugehörigkeit zu einer Minderheit. Die Frage der Wahrnehmung von Minderheitenrechten stellt sich erst danach, nämlich wenn die Zugehörigkeit zu einer Minderheit bereits bejaht worden ist. Rechtlich ausgedrückt betreffen Zusammengehörigkeits- oder Solidaritätsgefühl somit die Tatbestandsseite des Minderheitenbegriffs und nicht erst die Rechtsfolgenseite einer Minderheitenschutznorm. Zwarstehen grundsätzlich allen Minderheitenangehörigen ausnahmslos die Minderheitenrechte zu. Wer jedoch als Minderheitenangehöriger gilt, weil er alle Voraussetzungen der Minderheitendefinition erfüllt, hat dennoch das Recht, nicht als solcher behandelt (nicht: bezeichnet!!!) zu werden. Er kann also auf die ihm durch seinen Minderheitenstatus eigentlich zustehenden Minderheitenrechte verzichten. In diesem Sinne ist auch Art. 3 I des europäischen Rahmenabkommens zum Schutze nationaler Minderheiten zu verstehen. Darin heißt es: „Jede Person, die einer nationalen Minderheit angehört, hat das Recht, frei zu entscheiden, ob sie als solche behandelt werden möchte oder nicht [...]. ${ }^{\text {"102 }}$ Es wird dort also eine klare Trennung zwischen der faktischen Zugehörigkeit zu einer Minderheit („angehört") und der willentlich beeinflussbaren Behandlung als Minderheitenangehöriger („behandelt") vorgenommen. Die Entscheidung, als Minderheitenangehöriger behandelt zu werden und damit unter die besonderen Minderheitenschutzvorschriften zu fallen, ist erst Ausfluss der Minderheiteneigenschaft und somit unabhängig von der Entscheidung zum Bekenntnis zu einer (religiösen oder sprachlichen) Minderheit.

Dieses Ergebnis wird auch durch Ziffer 32 des Kopenhagener KSZE-Schlussdokuments vom 29.6.1990 bestätigt. Darin wird ausdrücklich erwähnt, dass „die Zugehörigkeit zu einer nationalen Minderheit [...] Angelegenheit der persönlichen Entscheidung eines Menschen " ${ }^{103}$ ist. Somit ist das subjektive Kriterium des Zusammengehörigkeits- bzw. Solidaritätsgefühls zumindest innerhalb Europas anerkanntes konstitutives Definitionsmerkmal einer Minderheit.

102 Art. 3 I des Rahmenabkommens zum Schutz nationaler Minderheiten vom 1.2.1995 (abgedruckt in: EuGRZ 1995: S. 269). Textliche Hervorhebungen des Autors.

103 Ziffer 32 des Dokuments des Kopenhagener Treffens vom 29.6.1990, in: Bulletin der Bundesregierung Nr. 88/1990: S. $757 \mathrm{ff}$. 


\subsubsection{Keine weiteren subjektiven Kriterien}

Das früher zum Teil vertretene Erfordernis der Loyalität ${ }^{104}$ zum Aufenthaltsstaat ist kein weiteres subjektives Kriterium der Minderheitendefinition. Denn selbst eine illoyale Minderheit bleibt Minderheit, wenn sie z. B. gewaltsam gegen die Mehrheit kämpfen sollte. Ob auch eine illoyale Minderheit in den Genuss uneingeschränkten Minderheitenschutzes kommen soll, betrifft nicht den Problemkreis der Minderheitendefinition, sondern denjenigen der konkreten Ausgestaltung des Minderheitenschutzes. ${ }^{105}$

\section{Minderheitenrechte in der EU}

Minderheitenrechte lassen sich ihrem Inhalt nach in negative und positive Minderheitenrechte einteilen. ${ }^{106}$ Negative Minderheitenrechte gewähren lediglich passiven Schutz (z. B. Nichtdiskriminierung) seitens des Staates, wohingegen positive Minderheitenrechte eine aktive Förderung der Minderheiten seitens des Staates zur Erreichung der rechtlichen und faktischen Gleichstellung der Minderheitenangehörigen mit den Angehörigen der Mehrheit sicherstellen sollen. ${ }^{107}$

Der Schutz von Minderheiten in der EU ergibt sich nur aus einem Zusammenwirken der Regelungen und Instrumente der drei europäischen Ebenen KSZE/OSZE, Europarat und EU. Diese sind durch einen gemeinsamen Bezugsrahmen eng miteinander verklammert. Hinzu kommen bilaterale völkerrechtliche Verträge zum Minderheitenschutz sowie Minderheitenschutzartikel im nationalen Verfassungsrecht der EU-Mitgliedsstaaten. ${ }^{108}$

104 Vgl. zum Loyalitätsbegriff: Luchterhandt, O. Nationale Minderheiten und Loyalität. Köln, 1997: S. $17 \mathrm{ff}$.

105 Vgl. Eglin, D. Demokratie und Minderheiten. Bern, 1998: S. 178; Ermacora, F. Nationale Minderheiten - Ein Definitionsproblem, in: Müller, K. (Hrsg.) Minderheiten im Konflikt. Zürich, 1993: S. 46; Kimminich, O. Rechtsprobleme der polyethnischen Staatsorganisation. Mainz, 1986: S. 100.

106 Vgl. Kurban, D. Confronting Equality: The Need for Constitutional Protection of Minorities on Turkey's Path to the European Union, in: Columbia Human Rights Law Review, Vol. 35, No. 1, 2003: S. 160 ff.; Oran, B. Türkiye'de Azınlıklar: Kavramlar, Lozan, İç Mevzuat, İçtihat, Uygulama. Istanbul, 2004: S. 32 ff. Eine in der Sache gleiche, jedoch begrifflich verschiedene Einteilung nimmt Krugmann vor, der zwischen Abwehr- und Leistungsrechten der Minderheitenangehörigen differenziert (vgl. Krugmann, M.: S. 216).

107 Vgl. Gornig, G. H. Die Definition des Minderheitenbegriffs aus historisch-völkischer Sicht: S. 20; Kurban, D. Confronting Equality: The Need for Constitutional Protection of Minorities on Turkey's Path to the European Union: S. 160 ff.; Oran, B. Türkiye'de Azınlıklar: Kavramlar, Lozan, İç Mevzuat, İçtihat, Uygulama: S. 32 ff.; Wheatley, S. Ulusal Azınlıkların Korunması Çerçeve Sözleşmesi, in: Kaboğlu, I. Ö. (Haz.) Azınlık Hakları. Istanbul, 2002: S. 193.

108 Vgl. Streinz, R.: S. 29. 
Gemäß Art. 6 II EU-Vertrag in der Fassung des Vertrages von Nizza vom 26.2.2001 ${ }^{109}$ hat die EU sowohl die Grundrechte $\mathrm{zu}$ achten, wie sie die EMRK gewährleistet, als auch diejenigen Grundrechte, wie sie sich aus den gemeinsamen Verfassungsüberlieferungen der Mitgliedsstaaten als allgemeine Grundsätze des Gemeinschaftsrechts ergeben. ${ }^{110}$ Laut Erklärung der EG-Außenminister zu den „Richtlinien für die Anerkennung neuer Staaten in Osteuropa und der Sowjetunion“ vom Dezember $1991^{111}$ sind auch die Garantien für die Rechte ethnischer und nationaler Minderheiten in Einklang mit den im Rahmen der KSZE eingegangenen Verpflichtungen $\mathrm{zu}$ beachten. Somit ergeben sich die Minderheitenrechte im Rahmen der EU aus den EU-Verträgen, der EU-Grundrechtecharta, der EMRK, den Dokumenten der KSZE sowie den gemeinsamen Verfassungsüberlieferungen der Mitgliedsstaaten. Im Einzelnen garantiert die EU demnach folgende negative wie auch positive Minderheitenrechte:

\subsection{Existenzrecht}

Als elementarste Form des Minderheitenschutzes finden sich in der EMRK, der EU-Grundrechtecharta und den nationalen Verfassungen der EU-Mitgliedsstaaten in unterschiedlichem Umfang Rechtsgewährungen, die das Recht auf Existenz der Gruppenangehörigen und damit auch der Gruppe selbst garantieren. ${ }^{112}$

\subsection{Diskriminierungsverbot}

Zentraler Bestandteil des europäischen Minderheitenschutzes ist das Diskriminierungsverbot. Dieses enthält das Verbot, aus Gründen der Minderheitenzugehörigkeit oder aus Gründen der Ausübung von Minderheitenrechten diskriminiert zu werden. $^{113}$

\subsection{Recht auf Gebrauch der eigenen Sprache}

Ein weiteres zentrales Minderheitenrecht ist das Recht auf Gebrauch der eigenen Sprache. Die umfassendsten Regelungen zum Gebrauch der Minderheitensprache

109 BGBl. 2001 II: S. 1666 ff.

110 Ein Beitritt der EG/EU zur EMRK ist nicht möglich, vgl. Gutachten des Europäischen Gerichtshofs vom 28.3.1996 (auszugsweise abgedruckt in: Europarecht 31 (1996), S. 302 ff.).

111 Richtlinien für die Anerkennung neuer Staaten in Osteuropa und in der Sowjetunion vom 16.12.1991, in: Europa-Archiv, Folge 2/1992, D 120.

112 Vgl. Art. 2 EMRK; Art. 2 EU-Grundrechtecharta.

113 Vgl. Art. 14 EMRK; Art. 21 EU-Grundrechtecharta; Ziffer 31 des Dokuments des Kopenhagener Treffens vom 29.6.1990; Ziffer I 1. der Charta von Paris vom 21.11.1990; Art. 2 der Antidiskriminierungsrichtlinie 2000/43/EG; Art. 2 der Gleichbehandlungsrahmenrichtlinie 2000/78/EG. 
finden sich in der Europäischen Charta für regionale und Minderheitensprachen. Darin wird ausführlich auf die verschiedenen Anwendungsgebiete (Gebrauch der Sprache im Unterricht, vor Behörden und Gerichten, im Bereich der Verwaltung und in den Medien) und auf die Bedeutung der Sprache bei kulturellen Ereignissen, in der Wirtschaft und der grenzüberschreitenden Zusammenarbeit eingegangen sowie werden entsprechende Maßnahmen zum Schutz und zur Förderung der Minderheitensprachen angeregt. ${ }^{114}$ Weiteren Niederschlag hat der Schutz der Minderheitensprache in den Diskriminierungsverboten der EMRK und der EU-Grundrechtecharta, dem Kopenhagener Schlussdokument sowie dem Rahmenübereinkommen zum Schutz nationaler Minderheiten gefunden. ${ }^{115}$

\subsection{Recht auf Bildung und Schule}

Das Recht auf Bildung und Schule ist eng mit dem Recht auf den Gebrauch der eigenen Sprache verbunden, da Schulunterricht in der Minderheitensprache eine wichtige Voraussetzung für den Erhalt der Minderheitensprache ist. Geregelt wird hier die Möglichkeit der schulischen und beruflichen Ausbildung unter besonderer Berücksichtigung der sprachlichen, geschichtlichen und kulturellen Besonderheiten der Minderheit. Soweit dies mit nationalem Recht vereinbar und auch finanzierbar ist, soll der Zugang zu staatlichen Schulen für alle Minderheitenangehörigen auf allen Ausbildungsstufen in der Muttersprache gewährleistet werden, in sprachlich gemischten Gebieten zweisprachiger Unterricht. ${ }^{116}$

\subsection{Kommunikationsrechte}

Die den Minderheitenangehörigen zustehenden Kommunikationsrechte umfassen das Recht auf ungehinderten Kontakt zu anderen Minderheitenangehörigen über die Staatsgrenzen hinaus ${ }^{117}$, das Recht auf ungehinderten Informationsaustausch in

114 Vgl. Art. 7-14 der Europäischen Charta für regionale und Minderheitensprachen.

115 Vgl. Art. 14 EMRK; Art. 21 EU-Grundrechtecharta; Ziffern 32.1 und 34 des Dokuments des Kopenhagener Treffens vom 29.6.1990; Art. 5, 9, 10, 11, 12, 14 des Rahmenübereinkommens zum Schutz nationaler Minderheiten.

116 Vgl. Art. 7 und 8 der Europäischen Charta für regionale und Minderheitensprachen; Ziffer 34 des Dokuments des Kopenhagener Treffens vom 29.6.1990; Art. 12, 13, 14 des Rahmenübereinkommens zum Schutz nationaler Minderheiten.

117 Vgl. Art. 10 EMRK; Art. 11 EU-Grundrechtecharta; Ziffer 32.4 des Dokuments des Kopenhagener Treffens vom 29.6.1990; Art. 9 und 17 des Rahmenübereinkommens zum Schutz nationaler Minderheiten. 
der Minderheitensprache ${ }^{118}$, das Recht auf ungehinderten Zugang zu innerstaatlichen Medien $^{119}$ sowie das Recht auf eigene Kommunikationsmittel ${ }^{120}$.

\subsection{Organisations- und Partizipationsrechte}

Organisations- und Partizipationsrechte der Minderheiten umfassen das Recht auf Gründung und Unterhaltung eigener Organisationen ${ }^{121}$ und das Recht auf politische Vertretung ${ }^{122}$. Eine wie auch immer geartete staatliche Förderung der minderheitlichen Organisations- und Partizipationsrechte sieht kein Dokument vor.

\subsection{Autonomierechte}

Die Möglichkeit, durch lokale oder autonome Verwaltungen die Identität von Minderheiten zu schützen und zu fördern, sieht lediglich das Kopenhagener Schlussdokument vor. Allerdings wird die Möglichkeit, lokale oder autonome Minderheitenverwaltungen einzurichten, dadurch eingeschränkt (und praktisch entwertet), dass dies in Einklang mit der Politik des betreffenden Staates stehen muss. ${ }^{123}$ Insofern besteht für Minderheiten faktisch kein eigenes Autonomierecht.

\subsection{Rechtsschutz}

Der Rechtsschutzals Zugang zu den Gerichten wird den Minderheitenangehörigen in der EU nur als Individualrecht gewährt. ${ }^{124}$ Eine besondere Berücksichtigung

118 Vgl. Art. 10 EMRK; Art. 11 EU-Grundrechtecharta; Ziffer 32.5 des Dokuments des Kopenhagener Treffens vom 29.6.1990; Ziffer 26 des Dokuments des Moskauer Treffens vom 3.10.1991; Art. 9 und 17 des Rahmenübereinkommens zum Schutz nationaler Minderheiten.

119 Vgl. Art. 10 EMRK; Art. 11 EU-Grundrechtecharta; Ziffer 32.5 des Dokuments des Kopenhagener Treffens vom 29.6.1990; Ziffer 26 des Dokuments des Moskauer Treffens vom 3.10.1991; Art. 11 der Europäischen Charta für regionale und Minderheitensprachen; Art. 9 des Rahmenübereinkommens zum Schutz nationaler Minderheiten.

120 Vgl. Art. 10 EMRK; Art. 11 EU-Grundrechtecharta; Ziffer 32.5 des Dokuments des Kopenhagener Treffens vom 29.6.1990; Art. 11 der Europäischen Charta für regionale und Minderheitensprachen; Art. 9 des Rahmenübereinkommens zum Schutz nationaler Minderheiten.

121 Vgl. Art. 11 EMRK; Art. 12 EU-Grundrechtecharta; Ziffern 32.2 und 32.6 des Dokuments des Kopenhagener Treffens vom 29.6.1990; Art. 7 und 8 des Rahmenübereinkommens zum Schutz nationaler Minderheiten.

122 Vgl. Ziffer 35 des Dokuments des Kopenhagener Treffens vom 29.6.1990; Art. 39 und 40 EUGrundrechtecharta.

123 Vgl. Ziffer 35 des Dokuments des Kopenhagener Treffens vom 29.6.1990.

124 Vgl. Art. 34 EMRK, Art. 47 EU-Grundrechtecharta. 
minderheitenspezifischer Belange findet sich lediglich hinsichtlich der Benutzung der Minderheitensprache vor Gericht. ${ }^{125}$

\section{FAZIT}

Obwohl es in der EU keine allgemein anerkannte und verbindliche Minderheitendefinition gibt, haben sich im politischen Prozess und in den Instanzen der EU einige regelmäßig aufgegriffene Merkmale herausgebildet, die - trotz fehlender Kodifikation - als konstante und konstituierende Kriterien eines europäischen Minderheitenbegriffs angesehen werden können. Dabei handelt es sich um die Kriterien der(1) zahlenmäßigen Unterlegenheit, der (2) nichtbeherrschenden Stellung, der (3) ethnischen, religiösen oder sprachlichen Eigenart, der (4) Staatsangehörigkeit des Aufenthaltsstaates sowie des (5) nach außen kundgetanen Zugehörigkeits- oder Solidaritätsgefühls. Konkret bedeutet dies, dass Minderheit nur eine Personengruppe sein kann, die (1) zahlenmäßig kleiner als der Rest der Bevölkerung ist, die (2) im Staat keine dominierende Rolle in Form von Alleinentscheidungsbefugnissen haben darf, die (3) mindestens eine ethnische, religiöse oder sprachliche Eigenart aufweisen muss, durch die sie sich vom Rest der Bevölkerung unterscheidet, deren Mitglieder (4) Staatsangehörige des Aufenthaltsstaates sein und (5) sich zu ihrer Personengruppe zugehörig und mit ihr solidarisch erklären müssen. Diese fünf Kriterien müssen jeweils kumulativ vorliegen, um eine Personengruppe als Minderheit qualifizieren zu können.

Der seit der Aufklärung politisch definierte europäische Minderheitenbegriff ist damit weit gefasst und schließt ethnische, nationale, religiöse und sprachliche Minderheiten ein. Somit stellt er eine große Anzahl von völkerrechtlich relevanten Gruppen unter besonderen Schutz und garantiert deren Angehörigen eine Vielzahl sich aus verschiedenen Dokumenten ergebender positiver wie negativer Minderheitenrechte. Um den Minderheitenschutz in der EU zu vereinheitlichen und damit noch effektiver zu gestalten, wäre es jedoch wünschenswert, den für die EU zugrunde liegenden Minderheitenbegriff im EU-Recht auch allgemein verbindlich zu kodifizieren. Hierzu fehlt gegenwärtig aber noch der politische Wille einiger Staaten wie Frankreich und Griechenland, die beispielsweise die Europäische Charta der regionalen und Minderheitensprachen von 1992 und das Rahmenübereinkommen des Europarates zum Schutz nationaler Minderheiten von 1995 entweder gar nicht unterzeichnet oder trotz Unterzeichnung nicht ratifiziert haben und sich damit aus auch für die EU wesentlichen Minderheitenschutzinstrumenten ausgeklinkt haben. Somit erscheinen einheitliche und für alle Mitgliedsstaaten verbindliche Minderheitenschutzstandards in der EU auch in Zukunft eher unwahrscheinlich zu sein.

125 Vgl. Art. 9 der Europäischen Charta für regionale und Minderheitensprachen; Ziffer 34 des Dokuments des Kopenhagener Treffens vom 29.6.1990. 


\section{Literaturverzeichnis}

Ahmed, T. The Impact of EU Law on Minority Rights. Oxford, 2011.

Berger, S. Europarat - Ein Auslaufmodell. Hamburg, 2008.

Berger, V. Rechtsprechung des Europäischen Gerichtshofs für Menschenrechte. Köln, 1987.

Bott-Bodenhausen, K. Die Bedeutung der Kultur für die Wahrung der Identität der Volksgruppe/Minderheit. In Blumenwitz, D.; Gornig, G. H. (Hrsg.) Minderheiten- und Volksgruppenrechte in Theorie und Praxis. Köln, 1993: 91-105.

Brunner, G. Die rechtliche Lage der Minderheiten in Mittel-, Ost- und Südosteuropa. In OstEuR 1994: 157 177.

Brunner, G. Nationalitätenprobleme und Minderheitenkonflikte in Osteuropa. Gütersloh, 1996.

Capotorti, F. Study on the Rights of Persons belonging to Ethnic, Religious and Linguistic Minorities. New York, 1991.

Çavuşoğlu, N. 2004 AB İlerleme Raporu ve Azınlık kavramı çerçevesinde Kültürel Haklara ilişkin sorunlar, Vortragsmanuskript zum gleichnamigen Vortrag auf der Konferenz der Istanbul Universität vom 8.12.2004 zum Thema „AB'ne Katılım Sürecinde İnsan Hakları".

Çavuşoğlu, N. Azınlık Hakları: Avrupa Standartlarıve Türkiye Bir Karşılaştırma. In Kaboğlu, I. Ö. (Haz.) Azınlık Hakları. Istanbul, 2002: 124-146.

Directorate-General for External Policies. Mainstreaming human and minority rights in the EU enlargement with the Western Balkans. Brussels, 2012.
Eglin, D. Demokratie und Minderheiten. Bern, 1998.

Ermacora, F. Nationale Minderheiten Ein Definitionsproblem. In Müller, K. (Hrsg.) Minderheiten im Konflikt. Zürich, 1993: 34-48.

Europäische Akademie Bozen. Package for Europe. Measures for Human Rights, Minority Protection, Cultural Diversity and Economic and Social Cohesion. Bozen, 1998.

Goldman, O. Q. The Need for an Independent International Mechanism to Protect Group Rights: A Case Study of the Kurds. In 2 Tulsa J. Comp. \& Int'l L. 45 (1994): 46-89.

Gornig, G. H. Die Definition des Minderheitenbegriffs aus historisch-völkischer Sicht. In Blumenwitz, D.; Gornig, G. H.; Murswiek, D. (Hrsg.) Ein Jahrhundert Minderheiten- und Volksgruppenschutz. Köln, 2001: 19-48.

Grabenwarter, C. Europäische Menschenrechtskonvention. München, 2003.

Hillgruber, C. Minderheitenschutz im Rahmen der Europäischen Menschenrechtskonvention - Stand und Entwicklung. In Blumenwitz, D.; Gornig, G. H. (Hrsg.) Minderheitenund Volksgruppenrecht in Theorie und Praxis. Köln, 1993: 39-47.

Hillgruber, C.; Jestaedt, M. Die Europäische Menschenrechtskonvention und der Schutz nationaler Minderheiten. Bonn, 1993.

Hofmann, R. Minderheitenschutz in Europa. Köln, 1995.

Kimminich, O. Rechtsprobleme der polyethnischen Staatsorganisation. Mainz, 1986. 
Kizilkan-Kisacik, Z. B. Europeanization of Minority Norms in Turkey. BadenBaden, 2013.

Klebes, H. Rechtsschutz von Minderheiten - Zu den Arbeiten des Europarats. In Blumenwitz, D.; Mangoldt, $\mathrm{H}$. von (Hrsg.) Fortentwicklung des Minderheitenschutzes und der Volksgruppenrechte in Europa. Köln, 1992: 47-60.

Klugmann, M. Europäische Menschenrechtskonvention und antiterroristische Maßnahmen. Frankfurt a. M., 2002.

Koplin, B. Nationale und ethnische Minderheiten im Verfassungsrecht der osteuropäischen Staaten. Berlin, 1995.

Krugmann, M. Das Recht der Minderheiten. Berlin, 2004.

Kurban, D. A Quest for Equality: Minorities in Turkey. Istanbul, 2007.

Kurban, D. Confronting Equality: The Need for Constitutional Protection of Minorities on Turkey's Path to the European Union. In Columbia Human Rights Law Review, Vol. 35, No. 1, 2003: 151-214.

Luchterhandt, O. Nationale Minderheiten und Loyalität. Köln, 1997.

Niewerth, J. Der kollektive und der positive Schutz von Minderheiten und ihre Durchsetzung im Völkerrecht. Berlin, 1996.

Nowak, M. UNO-Pakt über bürgerliche und politische Rechte und Fakultativprotokoll. In Nowak, M. CCPR-Kommentar. Kehl am Rhein, 1989.

Oeter, S. Minderheiten zwischen Segregation, Integration und Assimilation - Zur Entstehung und Entwicklung des Modells der Kulturautonomie. In Blumenwitz, D.; Gornig, G. H.; Murswiek, D. (Hrsg.) Ein Jahrhundert
Minderheiten- und Volksgruppenrechte. Köln, 2001: 63-81.

Oran, B. Küreselleşme ve Azınlıklar, 4. Basım. Ankara, 2001.

Oran, B. Lozan'ın „Azınlıkların Korunması" Bölümünü Yeniden Okurken. In SBF Dergisi, Cilt 49, No. 3-4 (1994): 283-301.

Oran, B. Türkiye'de Azınliklar: Kavramlar, Lozan, İç Mevzuat, İçtihat, Uygulama. Istanbul, 2004.

Pentassuglia, G. Minorities in international law. Strasbourg, 2002.

Peters, A. Einführung in die Europäische Menschenrechtskonvention. München, 2003.

Peukert, W. Neueste Entwicklungen des Menschenrechts- und Minderheitenschutzes - Übersicht über die Rechtsprechung des Europäischen Gerichtshofs für Menschenrechte. In Blumenwitz, D.; Gornig, G. H.; Murswiek, D. (Hrsg.) Fortschritte im Beitrittsprozess der Staaten Ostmittel-, Ost- und Südosteuropas zur Europäischen Union. Köln, 1999: 37-50.

Pogge, T. W. 'Group Rights and Ethnicity'. In Shapiro, I.; Kymlicka, W. (Eds.) Ethnicity and Group Rights. New York, 1997: 187-221.

Rehman, J. International Human Rights Law: A Practical Approach. Harlow, 2003.

Schmuck, O. Motive, Leitbilder und Etappen der Integration. In Bundeszentrale für politische Bildung (Hrsg.). Informationen zur politischen Bildung 279, Europäische Union. Bonn, 2003: 5-16.

Schumann, K. Der Stand der Arbeiten des Europarates zu Fragen nationaler Minderheiten. In Heuberger, V.; Suppan, A. (Hrsg.) Brennpunkt Osteuropa. München, 1996: 297-306. 
Schwellnus, G. Anti-discrimination legislation. In Rechel, B. (Ed.) Minority Rights in Central and Eastern Europe. London, 2009: 32-45.

Steiner, L. Die Entwicklung des Minderheitenschutzes im Rahmen des Europarates. In Blumenwitz, D.; Gornig, G. H. (Hrsg.) Minderheiten- und Volksgruppenrechte in Theorie und Praxis. Köln, 1993: 29-38.

Strasser, K. Grundrechtsschutz in Europa und der Beitritt der Europäischen Gemeinschaften zur Europäischen Menschenrechtskonvention. Frankfurt a. M., 2001.

Streinz, R. Minderheiten- und Volksgruppenrechte in der Europäischen Union. In Blumenwitz, D.; Gornig, G. H. (Hrsg.) Der Schutz von Minderheiten und Volksgruppen durch die Europäische Union. Köln, 1996: 11-29. Thornberry, P. International Law and the Rights of Minorities. Oxford, 1991.

Topidi, K. EU Law, Minorities and Enlargement. Antwerp, 2010.
Veiter, T. Wege zu einem modernen Volksgruppenrecht. In Aus Politik und Zeitgeschichte, B 18/1975: 29-38.

Vos, G. A. de. Ethnic Pluralism: Conflict and Accommodation - The Role of Ethnicity in Social History. In Romanucci-Ross, L.; Vos, G. A. de. Ethnic Identity: Creation, Conflict and Accommodation, $3^{\text {rd }}$ Ed. Walnut Creek, 1995: 15-47.

Wheatley, S. The Framework Convention for the Protection of Minorities. In EHRLR 6 (1996): 583-590.

Wheatley, S. Ulusal Azınlıkların Korunması Çerçeve Sözleşmesi. In Kaboğlu, I. Ö. (Haz.) Azınlık Hakları. Istanbul, 2002: 192-197.

White, N. D. The law of international organisations, 2nd Edition. Manchester, 2005.

Witte, B. de. The European Community and its Minorities. In Brölmann, C.; Lefeber, R.; Zieck, M. (Hrsg.) Peoples and Minorities in International Law. Dordrecht, 1993: 167-186.

\title{
EUROPIETIŠKOSIOS MAŽUMŲ KONCEPCIJOS RAIDA IR ITAKA II dalis
}

\author{
Arndt Künnecke \\ MEF universitetas, Turkija
}

Anotacija. Europoje neegzistuoja visuotinai pripažistamos tautiniu mažumu koncepcijos. Šio tyrimo pirmoje dalyje (publikuota šiuose mokslo darbuose, 2013, 20(4) analizuota mažumy koncepcijos formavimosi JT, UNESCO ir ESBO dokumentuose raida. Antroje šio tyrimo dalyje analizuojami specifiniai mažumu koncepcijos kriterijai, suformuoti su mažumu teisiu apsauga susijusiuose Europos Tarybos ir Europos Sajungos dokumentuose, kuriuos mažumu grupes Europoje turi atitikti, kad galètu naudotis minetu teisés aktu teikiama apsauga.

Reikšminiai žodžiai: tarptautine teisè, žmogaus teisès, mažumu teisès, mažumu samprata, Europos Sajunga. 


\title{
THE EUROPEAN CONCEPT OF MINORITIES. DEVELOPMENT AND IMPACT \\ Part II
}

\author{
Arndt Künnecke \\ MEF University Istanbul, Turkey
}

\begin{abstract}
Summary. Within the EU, the concept and rights of minorities play an important role, especially within the frame of the Copenhagen political criteria, which set the essential conditions that all candidate countries must satisfy to become a member state. According to these criteria, the candidate countries have to provide stability of institutions guaranteeing democracy, the rule of law, human rights and respect for and protection of minorities. How and according to which criteria minorities within the $E U$ are established and what kind of rights they have within the EU are examined in this essay, taking into account the historical development of the European concept of minorities and the content of the relevant documents in international law.

Even within the EU, a universally recognized and binding concept of minorities does not exist. To determine which minorities are supposed to be protected, some regularly applied characteristics have been developed within the political process and by some authorities of the EU. In spite of a lack of codification, these characteristics can be considered to be constant and continuous criteria of a European minority definition. These are the following criteria: (1) numerical inferiority, (2) non-dominant position, (3) distinctive ethnic, religious or linguistic characteristics, (4) citizenship of the country of residence and (5) explicitly made known sense of affiliation and solidarity. This means concretely that a minority can only be (1) a group of persons being numerically smaller than the rest of the population, (2) which may not have a dominant position in the state in the form of a sole right to make decisions, (3) which at least has to show a specific ethnic, religious or linguistic characteristic distinguishing them from the rest of the population, (4) members of which have to be citizens of their country of residence and (5) who have declared their membership and solidarity with their group of people. These five criteria have to be fulfilled cumulatively in order to be able to qualify a group of people as "minority".
\end{abstract}

Keywords: international law, human rights, minority rights, concept of minority, European Union.

Arndt Künnecke, MEF universiteto (Stambulas, Turkija) docentas, socialinių mokslų (teisè) daktaras. Mokslinių tyrimų kryptys: mažumų teisès, lyginamoji teisè.

Arndt Künnecke, MEF University (Istanbul, Turkey), Assist. Prof. Dr., lecturer. Research interests: minority rights, comparative law. 